Obere Extremität 2013 · 8:189

DOI 10.1007/s11678-013-0236-y

Online publiziert: 20. November 2013

(c) Springer-Verlag Berlin Heidelberg 2013

\title{
Chirurgie der Oberen Extremität - zwischen Schulter und Hand
}

Der/die spezielle Unfallchirurg(in) weil Fraktur? Der/die spezielle Orthopäde(in) - weil Prothese? Oder der/die Handchirurg(in) - weil N. ulnaris? Die meisten (winkel)stabilen Platten hat am ehesten der/die Unfallchirurg(in) eingesetzt, den meisten Zement rausgeholt der/die Orthopäde(in) und die meisten N.-ulnaris-Neurolysen der/die Handchirurg(in) (mit Lupenbrille). Vielleicht ist der/die durch die DVSE zertifizierte Schulter- und Ellenbogenchirurg(in) am besten geeignet?

Es gibt keine allgemeingültige Antwort auf die Frage, wer es machen soll - außer, dass es in einer Person nur wenige gibt, die wirklich maximale Erfahrung bei dieser komplexen Herausforderung haben. „Es sollte der(die)jenige machen, der/die es am besten kann“ - das ist klar - und wenn es passt, auch „die Besten gemeinsam“. Am Ellenbogengelenk kommen so Schulter- und Handchirurg(in) potenziell zusammen und das ist gut so (für den $\mathrm{Pa}$ tienten auf jeden Fall).

Um dieser Tendenz eine Struktur zu geben, wurde 2012 unter der Leitung von Lars Peter Müller (Köln), Sven Lichtenberg (Heidelberg) und Philip Kasten (Dresden) das Ellenbogenkomitee der DVSE gegründet. Eine Ellenbogenfellowship bei Graham King London Ontario wurde etabliert (2013: C. Gerhart/ Berlin), das Ellenbogenprothesenregister

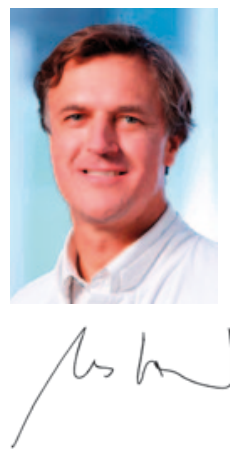

Markus Loew

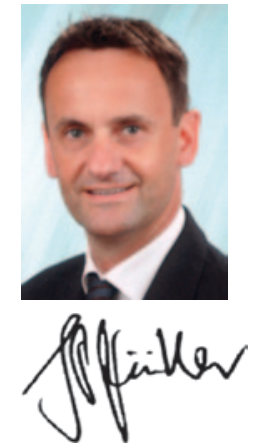

Lars Peter Müller wird vermutlich Ende diesen Jahres eröffnet werden (P. Magosch, Heidelberg und K. Burkhart, Köln). Versucht wird, die Aktivitäten der Ellenbogenkurse in Deutschland so zu koordinieren, dass vier „Kurse mit System“ die Voraussetzungen dafür schaffen, dass die theoretischen Anforderungen für den Erwerb des Ellenbogenzertifikates damit erfüllt werden können. In diesem Zusammenhang wurden die Weiterbildungsinhalte geändert und die Hälfte der geforderten Basisstunden zum Ellenbogen umverteilt. Ausdruck der hohen Aktivität ist auch der Ellenbogen„Pre-course“, der vor dem Jahreskongress der DVSE in Würzburg veranstaltet wurde und völlig ausgebucht war.

Eine Multicenter-Studie zu den Ursachen von Komplikationen nach Radiuskopffraktur hat begonnen. Ein großes klinisches Ziel der Gruppe ist die Standardisierung von Diagnostik und Therapie am Ellenbogengelenk, sodass bei Frakturen, Kontrakturen und Instabilitäten vorhersehbare Ergebnisse zu erzielen sind. Die Arthroskopie wird zunehmend an Bedeutung gewinnen, die rekonstruktiven Maßnahmen vermutlich mehr als die bis dato nur mittelfristig erfolgreichen prothetischen Maßnahmen.

Wir freuen uns auf die sicherlich interessante Entwicklung der Chirurgie an diesem Gelenk zwischen Schulter und Hand.

\section{Die Herausgeber}

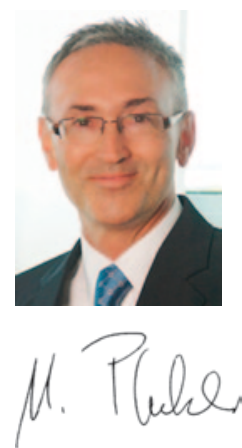

Manfred Pfahler
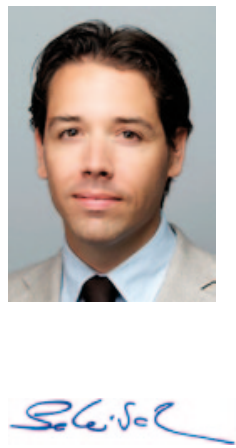

Markus Scheibel 